\title{
The Mathematical Representation of Turbulent Heat Fluxes Using Reynold's Decomposition
}

\author{
Kazeem 0. Rauff \\ Department of Physics, Federal University, Kashere, Nigeria \\ Email: rauffkazeem@fukashere.edu.ng
}

Received 8 January 2016; accepted 23 January 2016; published 27 January 2016

Copyright (C) 2016 by author and OALib.

This work is licensed under the Creative Commons Attribution International License (CC BY). http://creativecommons.org/licenses/by/4.0/

(c) (i) Open Access

\begin{abstract}
From a mathematical perspective, it is fundamental to develop a rigorous background upon which to study the physical quantities of a turbulent flow. The first problem in the mathematical theory is related to the deterministic nature of chaotic systems assumed in dynamical system theory and believed to hold inturbulence. This has actually not been proved for the Navier-Stokes equations. It is in fact one of the most outstanding open problems in mathematics to determine whether given an initial condition for the velocity field there exists, in some sense, a unique solution of the Navier-Stokes equations starting with this initial condition and valid for all later times. In a turbulent atmosphere, a turbulent stress term, the Reynolds stress, must be applied. All the terms in the horizontal motion equations are the order of $10^{-4}-10^{-3} \mathrm{~m} \cdot \mathrm{s}^{2}$. Under certain condition, some terms are very small and can be neglected for example, the rotational term is insignificant in the equation of vertical motion and has been omitted, instead gravitational acceleration term appears in the equation for vertical motion [1]; for steady flow, the tendency can be neglected; in the centre of high and low pressure, gradient force can be neglected; at the equator of for small scale processes, the Coriolis force can be neglected, and above the atmospheric boundary layer the stress terms can be neglected.
\end{abstract}

\section{Keywords}

Turbulent Heat Flux, Reynolds's Decomposition, Pressure Gradient Force, Sensible Heat Flux and the Latent Heat Flux

Subject Areas: Mathematical Analysis

\section{Introduction}

The earth's surface receives more radiation energy than that is lost. The surplus of supplied energy will be 
transported back to the atmosphere due to two turbulent energy fluxes, the sensible heat flux $\left(Q_{H}\right)$ and the latent heat flux ( $Q_{E}$, evaporation) [2]. The sensible heat flux is responsible for heating the atmosphere from the surface up to some $100 \mathrm{~m}$ during the day, except for days with strong convection [2]. The heat exchange in the air due to turbulence is much more effective. This is because turbulent exchange occurs over scales of motions ranging from millimeters to kilometers [2]. Turbulent elements can be thought of as air parcels with largely uniform thermodynamic characteristics. Small-scale turbulence elements join to form larger ones and so on. The largest eddies are atmospheric pressure systems. The heated turbulent elements transport their energy by their random motion. The larger turbulent elements receive their energy from the mean motion, and deliver the energy by a cascade process to smaller elements. Atmospheric turbulence is a specialty of the atmospheric motion consisting in the fact that air volume (much larger than molecules: turbulent elements, turbulent eddy) achieves irregular and stochastic motions around a mean state. They are of different order with characteristic extensions and lifetimes ranging from centimeters and seconds to thousands of kilometers and days [2]. The characteristic distribution of turbulent elements (turbulent eddies) takes place according to their size and is represented by the turbulence spectrum: The turbulence spectrum is a plot of the energy distribution of turbulent elements (turbulent eddies) according to their wavelength or frequency. Depending on the frequency, the distribution is classified as macro-, meso- or micro-turbulence [2]. Turbulence refers to the apparently chaotic nature of many flows, which is manifested in the form of irregular, almost random fluctuations in velocity, temperature, and scalar concentrations around their mean values in time and space [3].

The calculation of the heat fluxes (sensible and latent) caused by turbulent elements is analogous to ground heat flux using the vertical gradients of temperature $\mathrm{T}$ and specific humidity q respectively. The sensible heat flux, $Q_{H}$, describes the turbulent transport of heat from and to the earth's surface. The latent heat flux, $Q_{E}$, describes the vertical transport of water vapor and the heat required for evaporation at the ground surface.

\section{Methodology}

In the turbulent heat flow, the fluxes of flow variables are caused by differing properties of air parcels' moving in different directions relative to the mean flow (the mean flow usually being defined, from a practical perspective, as an average over a period of order an hour). For example if the rising air parcels are warmer that those descending, then there will be a vertical flux of heat $Q_{H}$. This heat flux, $Q_{H}$, is equal to $\rho c_{p}\left\langle w^{\prime} T^{\prime \prime}\right\rangle$, where $\rho$ and $c_{p}$ are the density and specific heat capacity of air and $\left\langle w^{\prime} T^{\prime \prime}\right\rangle$ is the covariance of $w^{\prime}$ and $T^{\prime \prime}$, the turbulent fluctuations in vertical velocity and in temperature about their means. Similarly, the covariance between the horizontal and vertical velocities gives rise to a downward vertical flux of horizontal momentum $\tau$ which is given by $-\rho\left\langle w^{\prime} u^{\prime}\right\rangle$, where $u^{\prime}$ is the horizontal component of velocity. Such a momentum flux is often referred to as a turbulent stress [4].

These turbulent fluxes have a major effect in the flow. For example, if one considers the average flow in a horizontally homogeneous boundary layer over an area of the Earth surface, the governing equation of the horizontal momentum balance is given by the Navier-Stokes equations [1] [3] [5] [6]:

$$
\begin{aligned}
& \frac{\partial u}{\partial t}=-u \frac{\partial u}{\partial x}-v \frac{\partial u}{\partial y}-w \frac{\partial u}{\partial z}-\frac{1}{\rho} \frac{\partial p}{\partial x}+f v+v \nabla^{2} u \\
& \frac{\partial v}{\partial t}=-u \frac{\partial v}{\partial x}-v \frac{\partial v}{\partial y}-w \frac{\partial v}{\partial z}-\frac{1}{\rho} \frac{\partial p}{\partial y}-f u+v \nabla^{2} v \\
& \frac{\partial w}{\partial t}=-u \frac{\partial w}{\partial x}-v \frac{\partial w}{\partial y}-w \frac{\partial w}{\partial z}+g+v \nabla^{2} w
\end{aligned}
$$

where $u$ is the horizontal wind in the $x$-direction (east); $v$ is the horizontal wind in the y-direction (north), and $w$ is the vertical wind; $p$ is the atmospheric pressure; $f$ is the Coriolis parameter; $g$ is the acceleration of gravity; $\rho$ is the air density; $v$ is the kinematic viscosity, and $\nabla^{2}$ is the Laplace operator. From left-to-right, the terms of the equation are the tendency, the advection, the pressure gradient force, the Coriolis force, and the (molecular) stress.

In a turbulent atmosphere, a turbulent stress term, the Reynolds stress, must be applied. All the terms in the horizontal motion equations are the order of $10^{-4}-10^{-3} \mathrm{~m} \cdot \mathrm{s}^{2}$. Under certain condition, some terms are very small and can be neglected for example, the rotational term is insignificant in the equation of vertical motion and 
has been omitted instead gravitational acceleration term appears in the equation for vertical motion [1]; for steady flow, the tendency can be neglected; in the centre of high and low pressure gradient force can be neglected; at the equator of for small scale processes the Coriolis force can be neglected, and above the atmospheric boundary layer the stress terms can be neglected.

\section{Results and Discussion}

The stress tensors are:

$$
\begin{aligned}
& P_{x x} P_{x y} P_{x z} \\
& P_{y x} P_{y y} P_{y z} \\
& P_{z x} P_{z y} P_{z z}
\end{aligned}
$$

The direct stresses are:

$$
\begin{aligned}
& P_{x x}=-P+2 \mu A+\lambda \Delta \\
& P_{y y}=-P+2 \mu B+\lambda \Delta \\
& P_{z z}=-P+2 \mu C+\lambda \Delta
\end{aligned}
$$

where

$$
A=\frac{\partial u}{\partial x}, B=\frac{\partial v}{\partial y}, C=\frac{\partial w}{\partial z}
$$

where $\lambda$ and $\mu$ (dynamic viscosity) are known as lame's constants, $\Delta$ is known as cubical dilatation and $v$ is kinematic viscosity.

$$
\lambda=\frac{-2}{3} \mu
$$

and

$$
v=\frac{\mu}{\rho}
$$

The shear stresses are given as:

$$
P_{x y}=P_{y x}=\mu\left(\frac{\partial u}{\partial y}+\frac{\partial v}{\partial x}\right)
$$

Under certain condition, some terms are very small and can be neglected for small scale processes the coriolis force can be neglected and above atmospheric boundary layer the stress terms can be neglected

The first terms of Equation (1) become:

$$
\frac{\partial u}{\partial t}=X+\frac{1}{\rho} \frac{\partial p}{\partial x}
$$

using Equation (2), we have:

$$
\frac{\partial u}{\partial t}=X+\frac{1}{\rho} \frac{\partial}{\partial x}\left(\frac{\partial P_{x x}}{\partial x}+\frac{\partial P_{x y}}{\partial y}+\frac{\partial P_{x z}}{\partial z}\right)
$$

Using from Equations (2), (5), and (6) in Equation (9) we have:

$$
\frac{\partial u}{\partial t}=X-\frac{1}{\rho} \frac{\partial P}{\partial x}+\mu \nabla^{2} u+\frac{\mu}{3 \rho} \frac{\partial \Delta}{\partial x}
$$

where the cubical dilatation, $\Delta$ is:

$$
\Delta=\nabla \cdot u=\nabla \cdot q
$$

Using (11) in (10), Equation (10) becomes: 


$$
\begin{aligned}
& \frac{\partial u}{\partial t}=X-\frac{1}{\rho} \frac{\partial P}{\partial x}+v \nabla^{2} u+\frac{\mu}{3 \rho} \frac{\partial}{\partial x} \nabla \cdot u \\
& \frac{\partial u}{\partial t}=X-\frac{1}{\rho} \frac{\partial P}{\partial x}+v \nabla^{2} u+\frac{\mu}{3 \rho} \nabla \nabla \cdot u \\
& \frac{\partial u}{\partial t}=X-\frac{1}{\rho} \frac{\partial P}{\partial x}+v \nabla^{2} u+\frac{v}{3} \nabla(\nabla \cdot u)
\end{aligned}
$$

from Maxwell identity, we have:

$$
\begin{aligned}
& \nabla \wedge(\nabla \wedge \nabla)=\nabla(\nabla \cdot u)-\nabla^{2} u \\
& \nabla^{2} u=\nabla(\nabla \cdot u)-\nabla \wedge(\nabla \wedge \nabla)
\end{aligned}
$$

putting (15) in (14) we have:

$$
\begin{gathered}
\frac{\partial u}{\partial t}=X-\frac{1}{\rho} \frac{\partial P}{\partial x}+\nu \nabla(\nabla \cdot q)-\nabla \wedge(\nabla \wedge \nabla)+\frac{v}{3} \nabla(\nabla \cdot u) \\
\frac{\partial u}{\partial t}=X-\frac{1}{\rho} \frac{\partial P}{\partial x}+\frac{4 v}{3} \nabla(\nabla \cdot u)-\nabla \wedge(\nabla \wedge u)
\end{gathered}
$$

\section{Reynolds's Decomposition}

This is the decomposition of all the variables into a mean part, $\bar{x}$, and a random fluctuating part, $x^{\prime}$. This is represented by:

$$
x=\bar{x}+x^{\prime}
$$

The application of Reynolds's decomposition requires some averaging rules for the turbulent values $x^{\prime}$, which are termed Reynolds's postulates:

$$
\begin{gathered}
\bar{c}=c \\
(\overline{c A})=c \bar{A} \\
(\overline{\bar{A} B})=\bar{A} \bar{B} \\
(\overline{A+B})=\bar{A}+\bar{B} \\
(\overline{\mathrm{d} A})=\frac{\mathrm{d} \overline{\mathrm{d}}}{\mathrm{d} t} \\
A=\bar{A}+A^{\prime} \quad \overline{\bar{A}}=\overline{\bar{A}+A^{\prime}}=\overline{\bar{A}}+\overline{A^{\prime}}=\bar{A} \\
\overline{B A^{\prime}}=\overline{\bar{B} \bar{A}}=0 \quad \overline{\bar{A}}=\overline{\left(\bar{A}+A^{\prime}\right)\left(\bar{B}+B^{\prime}\right)=\overline{\overline{A B}}+\bar{A} B^{\prime}+A^{\prime} \bar{B}+B^{\prime} A^{\prime}} \\
\overline{\bar{A} \bar{B}}+\overline{\bar{A} B^{\prime}}+\overline{A^{\prime} \bar{B}}+\overline{B^{\prime} A^{\prime}}=\bar{A} \bar{B}+\overline{B^{\prime} A^{\prime}}
\end{gathered}
$$

It is assumed that the postulates are universal, but for special spectral regions or for intermitted turbulence this is not valid [7]. The last postulate is the basis for the determination of turbulent fluxes according to the direct eddy covariance method. It is also assumed by [8] and [1]) that:

$$
\begin{aligned}
& \left|\frac{p^{\prime}}{\bar{p}}\right| \leq \leq\left|\frac{\rho^{\prime}}{\bar{\rho}}\right| \\
& \left|\frac{p^{\prime}}{\bar{p}}\right| \leq \leq\left|\frac{T^{\prime}}{\bar{T}}\right|
\end{aligned}
$$




$$
\left|\frac{\rho^{\prime}}{\bar{\rho}}\right| \leq \leq 1
$$

and

$$
\left|\frac{T^{\prime}}{\bar{T}}\right| \leq \leq 1
$$

In micrometeorology, we are interested in forecasting the mean quantities such as $\bar{u}$ and $\bar{T}$, while $u^{\prime}$ and $T^{\prime}$ are inherently random and the instantaneous values cannot be predicted. To determine the effect of the fluctuations on an equation we can replace each variable by the sum of its mean and its fluctuation and solve. So all occurrences of $x$ would be replaced using Equation (18). But by definition $\overline{u^{\prime}}=0$, no one might think that fluctuations average to zero. However, where we have the one fluctuating quantity multiplied by another is not the case. For example:

$$
\begin{aligned}
\overline{u w} & =\overline{\left(\bar{u}+u^{\prime}\right)\left(\bar{w}+w^{\prime}\right)}=\overline{\overline{u w}+\bar{u} w^{\prime}+u^{\prime} \bar{w}+w^{\prime} u^{\prime}} \\
& =\overline{\overline{u w}}+\overline{\bar{u} w^{\prime}}+\overline{u^{\prime} \bar{w}}+\overline{w^{\prime} u^{\prime}}=\overline{u w}+\overline{w^{\prime} u^{\prime}}
\end{aligned}
$$

When the average value of the product $u w$ is calculated, the terms $\bar{u} w^{\prime}$ and $u^{\prime} \bar{w}$ average out to zero, so:

$$
\begin{gathered}
u w=\left(\bar{u}+u^{\prime}\right)\left(\bar{w}+w^{\prime}\right)=\overline{u w}+\bar{u} w^{\prime}+u^{\prime} \bar{w}+w^{\prime} u^{\prime} \\
u w=\left(\bar{u}+u^{\prime}\right)\left(\bar{w}+w^{\prime}\right)=\overline{u w}+u^{\prime} w^{\prime}
\end{gathered}
$$

We are left with an extra term, the covariance of $u$ and $w$.

Applying Reynolds's decomposition to Equation (28) above we have:

$$
\begin{aligned}
& \frac{\partial \bar{u}}{\partial t}+\bar{u} \frac{\partial \bar{u}}{\partial x}+\bar{v} \frac{\partial \bar{u}}{\partial y}+\bar{w} \frac{\partial \bar{u}}{\partial z}+\frac{\partial}{\partial z}\left(\overline{u^{\prime} w^{\prime}}\right)=f v-\frac{1}{\rho} \frac{\partial \bar{P}}{\partial x} \\
& \frac{\partial \bar{v}}{\partial t}+\bar{u} \frac{\partial \bar{v}}{\partial x}+\bar{v} \frac{\partial \bar{v}}{\partial y}+\bar{w} \frac{\partial \bar{v}}{\partial z}+\frac{\partial}{\partial z}\left(\overline{v^{\prime} w^{\prime}}\right)=-f v-\frac{1}{\rho} \frac{\partial \bar{P}}{\partial y} \\
& \frac{\partial \bar{w}}{\partial t}+\bar{u} \frac{\partial \bar{w}}{\partial x}+\bar{v} \frac{\partial \bar{w}}{\partial y}+\bar{w} \frac{\partial \bar{w}}{\partial z}+\frac{\partial}{\partial z}\left(\overline{w^{\prime} w^{\prime}}\right)=g-\frac{1}{\rho} \frac{\partial \bar{P}}{\partial z}
\end{aligned}
$$

where:

$$
\frac{\partial}{\partial z}\left(\overline{u^{\prime} w^{\prime}}\right)=\frac{\partial}{\partial z}\left(\overline{v^{\prime} w^{\prime}}\right)=\frac{\partial}{\partial z}\left(\overline{w^{\prime} w^{\prime}}\right)=0
$$

The equation above is called continuity equation.

For all micrometeorological measurements steady state conditions are implied, $\frac{\partial}{\partial t}=0$, and a mostly homogeneous surface is necessary, $\frac{\partial}{\partial x_{i}}=0, \frac{\partial}{\partial x_{j}}=0$. Under these assumptions and including the components $u_{g}$ and $v_{g}$ of the geostrophic wind velocity and the angular velocity of the earth's rotation, $\Omega$, the three equations of motion in Equation (1) becomes [2]:

$$
\begin{gathered}
\frac{\partial \overline{u^{\prime} w^{\prime}}}{\partial z}=f\left(\bar{v}-\overline{v_{g}}\right)+v \nabla^{2} u, \overline{v_{g}}=\frac{1}{\rho f} \frac{\partial \bar{p}}{\partial x} \\
\frac{\partial \overline{v^{\prime} w^{\prime}}}{\partial z}=-f\left(\bar{u}-\overline{u_{g}}\right)+v \nabla^{2} v, \overline{u_{g}}=-\frac{1}{\rho f} \frac{\partial \bar{p}}{\partial y} \\
\frac{\partial{\overline{w^{\prime}}}^{2}}{\partial z}=\frac{1}{\rho} \frac{\overline{\partial p}}{\partial z}-g-2\left[\Omega_{u} \bar{v}-\Omega_{v} \bar{u}\right], f=2 \Omega \sin \varphi
\end{gathered}
$$

Equations (31) and (32) are the basis of the so-called ageostrophic method for the determination of the components of the shear stress tensor using differences between the wind velocity in the atmospheric boundary layer 
and the geostrophic wind [9] [10].

The gas law with the specific gas constant for dry air $R_{L}$ and the virtual temperature $T_{v}$ completes the system of equations [2]:

$$
p=\rho R_{L} T_{v}
$$

The covariance of the vertical wind velocity, $w$, and a horizontal wind component or a scalar $x$ can be determined by:

$$
\begin{aligned}
& \overline{w^{\prime} x^{\prime}}=\frac{1}{N-1} \sum_{k=0}^{N-1}\left[\left(w_{k}-\overline{w_{k}}\right)\left(x_{k}-\overline{x_{k}}\right)\right] \\
&=\frac{1}{N-1}\left[\sum_{k=0}^{N-1} w_{k} x_{k}-\frac{1}{N}\left(\sum_{k=0}^{N-1} w_{k} \sum_{k=0}^{N-1} x_{k}\right)\right] \\
& u_{*}^{2}=-\overline{u^{\prime} w^{\prime}}, \frac{Q_{H}}{\rho c_{p}}=\overline{T^{\prime} w^{\prime}}, \frac{Q_{E}}{\rho \lambda}=\overline{q^{\prime} w^{\prime}}, \frac{Q_{c}}{\rho}=\overline{c^{\prime} w^{\prime}}
\end{aligned}
$$

The friction velocity is a generalized velocity, i.e., it is the shear stress divided by the density

$$
\begin{gathered}
u_{*}=\left(\frac{\tau}{\rho}\right)^{1 / 2} \Rightarrow u_{*}^{2}=\rho \tau \\
u_{*}^{2}=\overline{u^{\prime} w^{\prime}}=K_{m} \frac{\partial \bar{u}}{\partial z}
\end{gathered}
$$

The new system of equations is similar to the Boussinesq system but is now valid for the average flow:

\section{Conclusion}

In a turbulent atmosphere, a turbulent stress term, the Reynolds stress, must be applied. All the terms in the horizontal motion equations are the order of $10^{-4}-10^{-3} \mathrm{~m} \cdot \mathrm{s}^{2}$. Despite the difficulties in the mathematical theory of the Navier Stoke Equation some successes have been collected such as estimates for the number of degrees of freedom in terms of fractal dimensions of suitable sets associated with the solutions of the Navier-Stokes equations, and partial estimates of a number of relations derived in the statistical theory of fully developed turbulence. It is concluded that in order to determine the effect of the fluctuations on an equation we can replace each variable by the sum of its mean and its fluctuation, and solve all occurrences of variables that would be replaced using Reynolds's postulate. But by definition $\overline{u^{\prime}}=0$, no one might think that fluctuations average to zero. However, where we have the one fluctuating quantity multiplied by another is not the case.

\section{References}

[1] Stull, R.B. (1988) An Introduction to Boundary Layer Meteorology. Kluwer Acad. Publ., Dordrecht, Boston, London, 666 p. http://dx.doi.org/10.1007/978-94-009-3027-8

[2] Foken, T. (2008) Micrometeorology. Springer-Verlag Berlin Heidelberg, 306 p.

[3] Arya, S.P. (2001) Introduction to Micrometeorology. Academic Press, San Diego, 415 p.

[4] Manson, P.J. and Thompson, D.J. (1992) Stochastic Backscatter in Large-Eddy Simulations of Boundary Layers. Journal of Fluid Mechanics, 242, 51-78. http://dx.doi.org/10.1017/S0022112092002271

[5] Etling, D. (2002) Theoretische Meteorologie. Springer, Berlin, Heidelberg, 354 p.

[6] Salby, M.L. (1995) Fundamentals of Atmospheric Physics. Academic Press, San Diego, New York, 624 p.

[7] Bernhardt, K. (1980) Zur Frage der Gültigkeit der Reynoldsschen Postulate. Z Meteorol, 30, 261-268.

[8] Businger, J.A. (1982) Equations and Concepts. In: Nieuwstadt, F.T.M. and Van Dop, H., Eds., Atmospheric Turbulence and Air Pollution Modelling: A Course Held in The Hague, 21-25 September 1981, D. Reidel Publ. Co., Dordrecht, 1-36.

[9] Bernhardt, K. (1970) Der ageostrophische Massenfluß in der Bodenreibungsschichtbeibeschleunigungsfreier Strömung. Z Meteorol, 21, 259-279.

[10] Lettau, H.H. (1957) Windprofil, innere Reibung und Energieumsatz in den untersten $500 \mathrm{~m}$ überdem Meer. Beitr Phys Atm, 30, 78-96. 